Note

\title{
Efficient synthesis of exomethylene- and keto-exomethylene-D-glucopyranosyl nucleoside analogs as potential cytotoxic agents
}

\author{
Niki Tzioumaki ${ }^{a}$, Evangelia Tsoukala ${ }^{a}$, Stella Manta ${ }^{a}$, Christos Kiritsis ${ }^{a}$, Jan Balzarini ${ }^{b}$, Dimitri Komiotis ${ }^{\mathrm{a}, *}$ \\ a Department of Biochemistry and Biotechnology, Laboratory of Organic Chemistry, University of Thessaly, 26 Ploutonos Str., 41221 Larissa, Greece \\ ${ }^{\mathrm{b}}$ Rega Institute for Medical Research, Katholieke Universiteit Leuven, B-3000 Leuven, Belgium
}

\section{A R T I C L E I N F O}

Article history:

Received 23 August 2010

Accepted 26 October 2010

Available online 30 October 2010

\section{Keywords:}

Unsaturated nucleosides

Exomethylene nucleosides

Ketonucleosides

Cytotoxicity

5-Fluorouracil

\begin{abstract}
A B S T R A C T
A novel series of exomethylene- and keto-exomethylene-D-glucopyranonucleosides with thymine, uracil, and 5-fluorouracil as heterocyclic bases have been designed and synthesized. Wittig condensation of the 3-keto glucoside 1 gave the corresponding 1,2:5,6-di-O-isopropylidene-3-deoxy-3-methylene-D-glucofuranose (2), which after hydrolysis and acetylation led to the precursor 1,2,4,6-tetra-O-acetyl-3-deoxy-3methylene-D-glucopyranose (4).Compound $\mathbf{4}$ was condensed with silylated thymine, uracil, and 5-fluorouracil, respectively, deacetylated and acetalated to afford 1-(3'-deoxy-4',6'-O-isopropylidene- $3^{\prime}$-methylene- $\beta$-D-glucopyranosyl)pyrimidines $\mathbf{7 a - c}$. Oxidation of the free hydroxyl group in the $2^{\prime}$-position of the sugar moiety led to the formation of the labile 1 -( $3^{\prime}$-deoxy- $4^{\prime}, 6^{\prime}-O$-isopropylidene- $3^{\prime}$-methylene- $\beta$-D-glucopyranosyl-2'-ulose)pyrimidines 8a-c. Finally, deisopropylidenation of the resulted derivatives 8a-c afforded the diol nucleosides 9a-c. The target keto-exomethylene analogs $\mathbf{9 a - c}$ were more cytostatic against a variety of tumor cell lines than the corresponding saturated-hydroxy-exomethylene derivatives 6. In particular, the 5 -fluorouracil derivative $9 \mathrm{c}$ was highly cytostatic at an $\mathrm{IC}_{50}$ ( $50 \%$ inhibitory concentration) ranging between 0.56 and $9.4 \mu \mathrm{g} / \mathrm{mL}$, which was comparable to the free parental 5-fluorouracil base.
\end{abstract}

(c) 2010 Elsevier Ltd. All rights reserved.
The number of modified nucleosides has grown almost exponentially in the last decades, at least in part in response to the pressing need for new antitumor and antiviral agents. ${ }^{1-4}$ While several modified nucleosides have clinically verified utility in the treatment of cancer ${ }^{5}$ and virus infections, such as HIV, ${ }^{6}$ both the demonstration of toxic side effects and the emergence of resistant viral strains stimulate further studies directed toward the synthesis and biology of modified nucleosides.

Lately, nucleosides containing pyranosyl rings have been synthesized along with their analogs in order to develop antitumor, ${ }^{7,8}$ antiviral, ${ }^{9-13}$ antioxidant, ${ }^{14}$ and antimicrobial reagents. ${ }^{15}$ Experimental data also revealed that human poly(A)-specific ribonuclease ${ }^{16}$ and glycogen phosphorylase ${ }^{17}$ are among the molecular targets of these compounds. Modifications in the sugar residue of the pyranonucleosides furnished enzymatically stable nucleic acid analogs, ${ }^{18,19}$ which can be considered as bioisosters of natural nucleosides and nucleotides, ${ }^{20}$ and can play a major role in different domains like therapy, diagnosis, and biotechnology.

In our continuing efforts to investigate new antiviral and antitumor agents, we have previously synthesized various unsaturated keto $^{21-24}$ or exomethylene nucleosides. ${ }^{25-27}$ These uncommon pyranonucleosides proved to have a promising potential in combating rotaviral infections and exhibited cytostatic activity against various

\footnotetext{
* Corresponding author. Tel.: +30 2410 565285; fax: +30 2410565290

E-mail address: dkom@bio.uth.gr (D. Komiotis).
}

cancer cell lines. Experimental evidence also suggested that these agents may act as novel types of 5-fluorouracil releasing prodrugs $^{28}$ that target thymidylate synthase for their cytostatic action.

With the above applications in mind and as continuation of our work in the synthesis of biologically active nucleosides, the aim of the present study is the stereocontrolled synthesis of pyranonucleosides, where we combined both the pharmacologically active keto group and exomethylene moiety in the sugar part to obtain new informations about structure-activity relationships. Therefore, we report herein the synthesis of a novel class of enone glucopyranonucleosides possessing an exomethylene group in the $3^{\prime}$-position and a keto group in the $2^{\prime}$-position of the sugar portion, containing thymine, uracil, and 5-fluorouracil as base moieties.

Retrosynthetic analysis suggested that the $3^{\prime}$-methylene nucleosides could be obtained from the correspoding $3^{\prime}$-keto nucleosides after Wittig olefination. However, the instability of the $3^{\prime}$-keto nucleosides, due to their susceptibility to $\beta$-elimination of the nucleobase $^{29,30}$ prompted us to choose the appropriate 3-keto glucoside as the suitable precursor.

3'-Methylene-2'-keto- $\beta$-D-glucopyranonucleosides 9a-c were prepared according to the synthetic route outlined in Scheme 1. Wittig condensation of the 1,2:5,6-di-O-isopropylidene- $\alpha$-D-ribo-hexofuranos-3-ulose $(\mathbf{1})^{31}$ with $\mathrm{NaH}$ and methyl triphenylphosphonium bromide $\left(\mathrm{Ph}_{3} \mathrm{P}^{+} \mathrm{CH}_{3} \mathrm{Br}^{-}\right)$at $0{ }^{\circ} \mathrm{C}$, in the presence of $t$-amyl alcohol ${ }^{32}$ in tetrahydrofuran (THF), led to the formation of the desired 1,2:5,6-di$O$-isopropylidene-3-deoxy-3-methylene-D-glucofuranose $(2),{ }^{33}$ in 


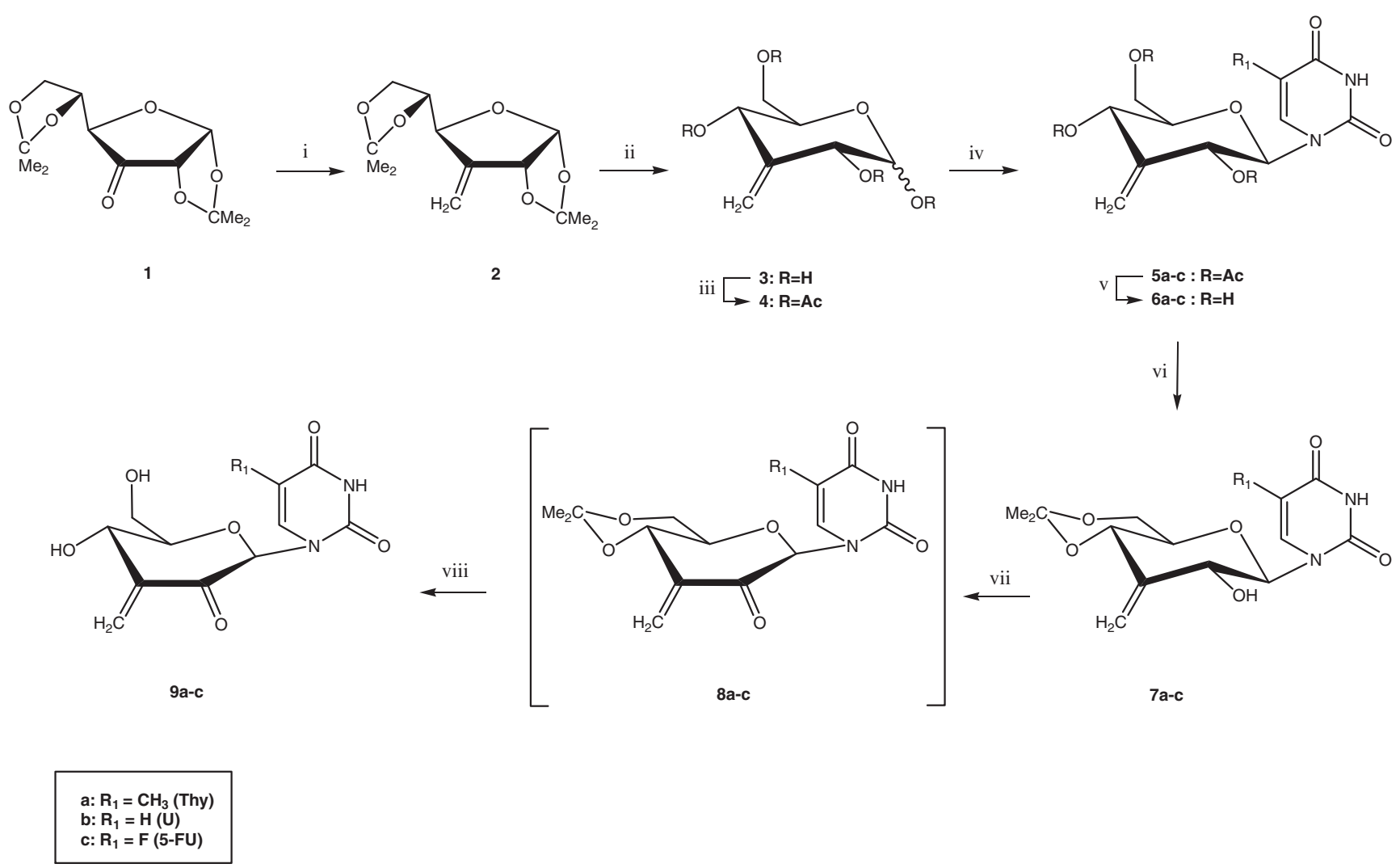

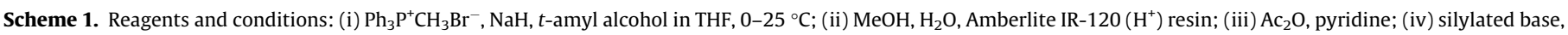

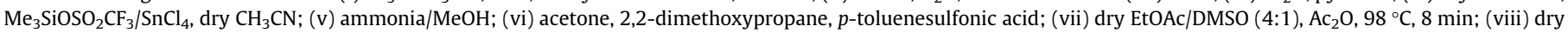
$\mathrm{CH}_{2} \mathrm{Cl}_{2}$, methanolic hydrogen chloride.

$75 \%$ yield. Hydrolysis of 2 using Amberlite IR $120\left(\mathrm{H}^{+}\right)$resin in methanol followed by acetylation using acetic anhydride $\left(\mathrm{Ac}_{2} \mathrm{O}\right)$ in pyridine $^{31}$ led to the 1,2,4,6-tetra-O-acetyl-3-deoxy-3-methylene-Dglucopyranose (4).

The protected, 1-(2',4',6'-tri-O-acetyl-3'-deoxy-3'-methylene$\beta$-D-glucopyranosyl) nucleosides of thymine (5a), uracil (5b) and 5 -fluorouracil $(\mathbf{5 c})$, were obtained upon condensation of the precursor material $\mathbf{4}$ with silylated thymine, uracil and 5-fluorouracil, respectively, in the presence of tin chloride(IV) and trimethylsilyl trifluomethane-sulfonate $\left(\mathrm{Me}_{3} \mathrm{SiOSO}_{2} \mathrm{CF}_{3}\right)$ in refluxing acetonitrile. ${ }^{34}$ Removal of all $O$-acetyl protecting groups of $\mathbf{5 a - c}$, with saturated methanolic ammonia, ${ }^{35}$ gave $\mathbf{6 a - c}$ in excellent yields. Specific acetalation of the aforementioned compounds using 2,2dimethoxypropane ${ }^{35}$ in acetone led to the $2^{\prime}, 3^{\prime}$-O-isopropylidene derivatives $7 \mathbf{a}-\mathbf{c}$, in yields $72 \%, 75 \%$, and $68 \%$, respectively. The ${ }^{1} \mathrm{H}$ NMR spectra of $\mathbf{7 a - c}$ showed the presence of two vinylic protons at 5.40-5.34 ppm and 5.35-5.32 ppm, each as a broad singlet, corresponding to the $3^{\prime}$-exomethylene groups.

The crucial step of the synthetic pathway proved to be the oxidation of the suitably protected precursors $7 \mathbf{a}-\mathbf{c}$. Therefore, oxidation of 7a-c with the Pfitzner and Moffatt ${ }^{36}$ [dimethyl sulfoxide (DMSO)/ $N, N^{\prime}$-dicyclohexylcarbodiimide (DCC)], ${ }^{37,38}$ Garegg and Samuelsson ${ }^{39}\left(\mathrm{CrO}_{3} /\right.$ pyridine/ $\left.\mathrm{Ac}_{2} \mathrm{O}\right),{ }^{40}$ Swern $^{41}$ (DMSO/oxalyl chloride), ${ }^{42,43}$ and pyridinium dichromate (PDC)/3E molecular sieves ${ }^{44}$ reagents resulted in $\beta$-elimination of the nucleobase, while a mixture of untreatable products was obtained. Dess-Martin ${ }^{45}$ (12-I-5-periodinane) oxidation ${ }^{30}$ of $\mathbf{7 a - c}$ afforded the desired methylene ketonucleosides $\mathbf{8 a - c}$ but unfortunately they could not be separated from the iodo-compound byproduct.

However, oxidation performed by a modified Albright-Goldman method using the DMSO/EtOAc/ $/ \mathrm{Ac}_{2} \mathrm{O}$ system ${ }^{46}$ led to the target 1-(3'-deoxy-4',6'-O-isopropylidene-3'-methylene- $\beta$-D-glucopyranosyl-2'-ulose)nucleosides $\mathbf{8 a}-\mathbf{c}$, well characterized by ${ }^{1} \mathrm{H}$ NMR and mass spectroscopy. Even though the desired compounds were readily available after isolation, they seemed to be labile during storage. Therefore, they were immediately treated with methanolic hydrogen chloride ${ }^{47}$ to give the diol nucleosides 9a-c, in yields $63 \%, 60 \%$, and $55 \%$, respectively. It should be mentioned that treatment of $\mathbf{8 a - c}$ with a mixture of $\mathrm{CH}_{2} \mathrm{Cl}_{2} / \mathrm{HCOOH}$, $1: 1$, or $90 \%$ trifluoroacetic acid in methanol $(\mathrm{MeOH})$, or pyridinium p-toluenesulfonate (PPTS) in $\mathrm{MeOH},{ }^{48}$ or Amberlite IR $120\left(\mathrm{H}^{+}\right)$re$\sin ,{ }^{49}$ or $p$-toluenesulfonic acid in $\mathrm{MeOH}^{50}$ unfortunately did not afford the target nucleosides $\mathbf{9 a - c}$ but it led instead to intractable materials.

The target nucleosides 9a-c and their key intermediates saturated exomethylene 6a-c were tested for their inhibitory effects on the proliferation of murine leukemia cells (L1210), human T-lymphocyte cells (CEM), and human cervix carcinoma cells (HeLa).

In contrast to the previously synthesized saturated 2 '- or 4 '-exomethylene nucleosides, ${ }^{25-27}$ no marked cytostatic activity was noticed for the newly $3^{\prime}$-exomethylene analogs $\mathbf{6 a - c}$ (Table 1). It appears that the transposition of the methylene moiety from $\mathrm{C}-2^{\prime}$ or $\mathrm{C}-4^{\prime}$ to $\mathrm{C}-3^{\prime}$ lowers the cytotoxic activity of this type of molecules. On the other hand, the target keto-exomethylene derivatives $9 a-c$ were endowed with an antiproliferative activity, in particular the 5-fluorouracil derivative 9c, indicating the important role of the keto group in the eventual cytostatic activity of these compounds. Compound 9c showed a very similar cytostatic activity as the free base 5-fluorouracil (5-FU), and therefore, we assume that the compound acts as an efficient prodrug of 5-FU. Our results enforce the importance of the presence of the keto-group together 
Table 1

Inhibitory effects on the proliferation of murine leukemia cells (L1210), human T-lymphocyte cells (CEM), and human cervix carcinoma cells (HeLa)

\begin{tabular}{llll}
\hline Compound & \multicolumn{3}{c}{$\mathrm{IC}_{50}{ }^{\mathrm{a}}(\mu \mathrm{g} / \mathrm{ml})$} \\
\cline { 2 - 4 } & $\mathrm{L} 1210$ & $\mathrm{CEM}$ & HeLa \\
\hline $\mathbf{6 a}$ & $>200$ & $>200$ & $>200$ \\
$\mathbf{9 a}$ & $77 \pm 24$ & $126 \pm 34$ & 73 \\
$\mathbf{6 b}$ & $>200$ & $>200$ & $>200$ \\
$\mathbf{9 b}$ & $84 \pm 10$ & $101 \pm 14$ & 92 \\
$\mathbf{6 c}$ & $>200$ & $>200$ & $>200$ \\
$\mathbf{9 c}$ & $0.56 \pm 0.21$ & $9.4 \pm 3.0$ & $1.5 \pm 0.0$ \\
5 -Fluorouracil & $0.49 \pm 0.13$ & $18 \pm 5$ & $0.54 \pm 0.12$ \\
\hline
\end{tabular}

a $50 \%$ Inhibitory concentration or compound concentration required to inhibit tumor cell proliferation by $50 \%$.

with the carbon-carbon double bond in the sugar moiety of the nucleoside analogs. This conjugation proved to be once again a prerequisite for biological activity. ${ }^{21-28}$ It appears that the unsaturated keto system might function as an acceptor in a Michael-addition type mechanism. ${ }^{27}$

\section{Experimental}

\subsection{General procedure}

Melting points were recorded in a Mel-Temp apparatus and are uncorrected. Thin-layer chromatography (TLC) was performed on Merck precoated $60 \mathrm{~F}_{254}$ plates. Reactions were monitored by TLC on silica gel, with detection by UV light $(254 \mathrm{~nm})$ or by charring with sulfuric acid. Flash chromatography was performed using silica gel (240-400 mesh, Merck). ${ }^{1} \mathrm{H}$ and ${ }^{13} \mathrm{C}$ NMR spectra were obtained at room temperature with a Bruker 400 spectrometer at 400 and $100 \mathrm{MHz}$, respectively, using chloroform- $d\left(\mathrm{CDCl}_{3}\right)$, methanol$d_{4}\left(\mathrm{CD}_{3} \mathrm{OD}\right)$, and acetone- $d_{6}\left(\left(\mathrm{CD}_{3}\right)_{2} \mathrm{CO}\right)$. Chemical shifts are reported in parts per million $(\delta)$ downfield from tetramethylsilane (TMS) as internal standard. Mass spectra were obtained with a Micromass Platform LC (ESIMS). Optical rotations were measured using Autopol I polarimeter.

All reactions were carried out in dry solvents. $\mathrm{CH}_{2} \mathrm{Cl}_{2}$ was distilled from phosphorous pentoxide and stored over $4 \mathrm{E}$ molecular sieves. Acetonitrile and toluene (Tol) were distilled from calcium hydride and stored over 3E molecular sieves. THF was freshly distilled under nitrogen from sodium/benzophenone before use and pyridine stored over pellets of potassium hydroxide.

\subsection{Synthesis of 1,2,4,6-tetra-O-acetyl-3-deoxy-3-methylene-D- glucopyranose (4)}

\subsubsection{1,2:5,6-Di-0-isopropylidene-3-deoxy-3-methylene-D- glucofuranose (2)}

To a stirred suspension of $\mathrm{Ph}_{3} \mathrm{P}^{+} \mathrm{CH}_{3} \mathrm{Br}^{-}$(54.48 mmol, $19.50 \mathrm{~g}$ ) and $t$-amyl alcohol $(59.44 \mathrm{mmol}, 6.51 \mathrm{~mL})$ in dry THF $(268.11 \mathrm{~mL})$ was added $\mathrm{NaH}\left(95.10 \mathrm{mmol}, 2.28 \mathrm{~g}, 60 \%\right.$ in oil) at $0{ }^{\circ} \mathrm{C}$ and the reaction mixture was stirred for $2 \mathrm{~h}$ at ambient temperature under nitrogen. To this yellow phosphorous ylide was added a solution of 1,2:5,6-di-O-isopropylidene- $\alpha$-D-ribo-hexofuranos-3-ulose $(\mathbf{1})^{31}$ $(16.51 \mathrm{mmol}, 4.26 \mathrm{~g})$ in dry THF $(26.28 \mathrm{~mL})$ dropwise, at $0{ }^{\circ} \mathrm{C}$ under nitrogen. After the mixture was stirred for $30 \mathrm{~min}$ at ambient temperature, the reaction mixture was quenched with saturated sodium bicarbonate and extracted with EtOAc. The organic layer was washed with water, dried with sodium sulfate, and evaporated. The residue was purified by flash chromatography (hexane, $R_{\mathrm{f}} 0.2$ ) to give $3.17 \mathrm{~g}(75 \%)$ of compound 2 as a white solid: $\mathrm{mp}$ $85-87^{\circ} \mathrm{C}$ (hexane-EtOAc); $[\alpha]_{\mathrm{D}}^{22}+2\left(\mathrm{c} 0.3, \mathrm{CHCl}_{3}\right)$; The ${ }^{1} \mathrm{H}$ NMR data agreed with the assignments ${ }^{51}$ corrected from the initial report; ${ }^{52}$ Anal. Calcd for $\mathrm{C}_{13} \mathrm{H}_{20} \mathrm{O}_{5}$ : C, 60.92; $\mathrm{H}, 7.87$. Found: C, 60.98; $\mathrm{H}, 7.83$. ESIMS: $m / z 257.3\left[\mathrm{M}+\mathrm{H}^{+}\right]$.

\subsubsection{3-Deoxy-3-methylene-D-glucopyranose (3)}

To a solution of $2(12.38 \mathrm{mmol}, 3.17 \mathrm{~g})$ in $\mathrm{MeOH}(19.5 \mathrm{~mL})$ and $\mathrm{H}_{2} \mathrm{O}(110.6 \mathrm{~mL})$ was added Amberlite IR $120\left(\mathrm{H}^{+}\right)$resin and the mixture was refluxed overnight. The reaction mixture was filtered and evaporated to dryness to give $1.85 \mathrm{~g}(85 \%)$ of compound 3 as a viscous oil and it was used without further purification (1:9 $\mathrm{MeOH}-\mathrm{EtOAc}, R_{\mathrm{f}} 0.4$ ): $[\alpha]_{\mathrm{D}}^{22}-8$ (c $\left.0.5, \mathrm{MeOH}\right) ;$ Anal. Calcd for $\mathrm{C}_{7} \mathrm{H}_{12} \mathrm{O}_{5}$ : C, 47.72; $\mathrm{H}, 6.87$. Found: $\mathrm{C}, 47.86 ; \mathrm{H}, 6.82$. ESIMS: $\mathrm{m} / \mathrm{z}$ $177.2\left[\mathrm{M}+\mathrm{H}^{+}\right]$

\subsubsection{1,2,4,6-Tetra-0-acetyl-3-deoxy-3-methylene-D- glucopyranose (4)}

Compound 3 (10.53 mmol, $1.85 \mathrm{~g}$ ) was dissolved in a mixture of pyridine $(37.1 \mathrm{~mL})$ and $\mathrm{Ac}_{2} \mathrm{O}(19.4 \mathrm{~mL})$. The reaction was carried out at room temperature for $1 \mathrm{~h}$, then was quenched with $\mathrm{MeOH}$ at $0{ }^{\circ} \mathrm{C}$, and was concentrated in vacuum. The residue was diluted with EtOAc and washed with saturated sodium bisulfate, sodium bicarbonate, and water. The organic extract was dried over anhydrous sodium sulfate, filtered, and evaporated to dryness to give $3.55 \mathrm{~g}$ (98\%) of tetraacetylated 4 , $\left(0.5: 9.5 \mathrm{MeOH}\right.$-EtOAc, $\left.R_{\mathrm{f}} 0.54\right)$ as a white foam: $[\alpha]_{\mathrm{D}}^{22}+10\left(\mathrm{c} 0.5, \mathrm{CHCl}_{3}\right)$; Anal. Calcd for $\mathrm{C}_{15} \mathrm{H}_{20} \mathrm{O}_{9}$ : C, 52.32; $\mathrm{H}, 5.85$. Found: $\mathrm{C}, 52.36 ; \mathrm{H}, 5.92$. ESIMS: $m / z$ $345.3\left[\mathrm{M}+\mathrm{H}^{+}\right]$.

\subsection{Synthesis of 1-(3'-deoxy-3'-methylene- $\beta$-D-glucopyranosyl- $2^{\prime}$-ulose)thymine (9a)}

\subsubsection{1-(2',4',6'-Tri-0 -acetyl-3'-deoxy-3'-methylene- $\beta$-D- glucopyranosyl)thymine ( $5 a)$}

A mixture of thymine ( $14.45 \mathrm{mmol}, 1.82 \mathrm{~g})$, hexamethyldisilazane (HMDS) (17.92 mmol, $3.78 \mathrm{~mL})$, and saccharin $(0.66 \mathrm{mmol}$, $121.76 \mathrm{mg})$ in dry $\mathrm{CH}_{3} \mathrm{CN}(47 \mathrm{~mL})$ was refluxed for 30 min under nitrogen. To this were added 1,2,4,6-tetra-O-acetyl-3-deoxy-3methylene-D-glucopyranose (4) (10.32 mmol, $3.55 \mathrm{~g}$ ) and $\mathrm{SnCl}_{4}$ $(14.45 \mathrm{mmol}, 1.7 \mathrm{~mL})$. The reaction mixture was refluxed for $1 \mathrm{~h}$, neutralized with saturated sodium bicarbonate, and then extracted with $\mathrm{CH}_{2} \mathrm{Cl}_{2}$. The organic extract was dried over anhydrous sodium sulfate, filtered, and evaporated to dryness. The residue was purified by flash chromatography (4:6 hexane-EtOAc, $R_{\mathrm{f}} 0.4$ ) to give $2.33 \mathrm{~g}(55 \%)$ of compound $\mathbf{5 a}$ as a white solid: $\mathrm{mp} 102-104{ }^{\circ} \mathrm{C}$ (hexane-EtOAc); $[\alpha]_{\mathrm{D}}^{22}-2\left(c \quad 0.5, \mathrm{CHCl}_{3}\right) ; \lambda_{\max }\left(\mathrm{CHCl}_{3}\right) 263 \mathrm{~nm}(\varepsilon$ 8502); ${ }^{1} \mathrm{H}$ NMR $\left(\mathrm{CDCl}_{3}, 400 \mathrm{MHz}\right): \delta 8.17$ (br s, $\left.1 \mathrm{H}, \mathrm{NH}\right), 7.19$ (s, $1 \mathrm{H}, \mathrm{H}-6), 5.67$ (d, $\left.1 \mathrm{H}, J_{1^{\prime}, 2^{\prime}} 9.3 \mathrm{~Hz}, \mathrm{H}-1^{\prime}\right), 5.50$ (d, $\left.1 \mathrm{H}, \mathrm{H}-2^{\prime}\right), 5.37$ (d, $\left.1 \mathrm{H}, J_{4^{\prime}, 5^{\prime}} 9.6 \mathrm{~Hz}, \mathrm{H}-4^{\prime}\right), 5.21,5.12(2 \mathrm{~s}, 2 \mathrm{H}$, methylene), 4.344.15 ( $\left.\mathrm{m}, 2 \mathrm{H}, \mathrm{H}-6 \mathrm{a}^{\prime}, \mathrm{H}-6 \mathrm{~b}^{\prime}\right), 3.80-3.75\left(\mathrm{~m}, 1 \mathrm{H}, \mathrm{H}-5^{\prime}\right), 2.19,2.11$, $2.10(3 \mathrm{~s}, 9 \mathrm{H}, 3 \mathrm{OAc}), 1.96\left(\mathrm{~s}, 3 \mathrm{H}, 5-\mathrm{CH}_{3}\right)$. Anal. Calcd for $\mathrm{C}_{18} \mathrm{H}_{22} \mathrm{~N}_{2} \mathrm{O}_{9}$ : C, 52.68; H, 5.40; N, 6.83. Found: C, 52.73; H, 5.34; $\mathrm{N}, 6.82$. ESIMS $m / z 411.4\left[\mathrm{M}+\mathrm{H}^{+}\right]$.

\subsubsection{1-(3'-Deoxy-3'-methylene- $\beta$-D-glucopyranosyl)thymine (6a)}

Compound 5a (5.67 mmol, $2.33 \mathrm{~g}$ ) was treated with ammonia/ $\mathrm{MeOH}$ (saturated at $0{ }^{\circ} \mathrm{C}, 240 \mathrm{~mL}$ ). The solution was stirred overnight at room temperature and then was concentrated under reduced pressure. The residue was purified by flash chromatography (2:8 MeOH-EtOAc, $R_{\mathrm{f}} 0.45$ ) to give $1.55 \mathrm{~g}$ (96\%) of compound 6a, as a white foam: $[\alpha]_{\mathrm{D}}^{22}-2(c 0.3, \mathrm{MeOH}) ; \lambda_{\max }(\mathrm{MeOH}) 263 \mathrm{~nm}$ ( $\varepsilon$ 6322); ${ }^{1} \mathrm{H}$ NMR $\left(\mathrm{CD}_{3} \mathrm{OD}, 400 \mathrm{MHz}\right): \delta 7.65(\mathrm{~s}, 1 \mathrm{H}, \mathrm{H}-6), 5.39(\mathrm{~s}$, 2H, methylene), 5.36 (d, 1H, J ${1^{\prime}, 2^{\prime}}^{\prime} 9.2 \mathrm{~Hz}, \mathrm{H}-1^{\prime}$ ), 4.26 (d, 1H, H-2'), 4.12 (d, 1H, $\left.J_{4^{\prime}, 5^{\prime}} 9.6 \mathrm{~Hz}, \mathrm{H}-4^{\prime}\right), 3.92-3.79$ (m, 2H, H-6a', H-6b'), 3.43-3.29 (m, 1H, H-5'), $1.93\left(\mathrm{~s}, 3 \mathrm{H}, 5-\mathrm{CH}_{3}\right) ;{ }^{13} \mathrm{C} \mathrm{NMR}\left(\mathrm{CD}_{3} \mathrm{OD}\right.$, $100 \mathrm{MHz}): 152.0,150.5,149.0,137.2,110.5,104.8,85.1,82.5$, 69.5, 67.2, 61.3, 10.9. Anal. Calcd for $\mathrm{C}_{12} \mathrm{H}_{16} \mathrm{~N}_{2} \mathrm{O}_{6}$ : C, 50.70; $\mathrm{H}$, 5.67; N, 9.85. Found: C, 50.91; H, 5.59; N, 9.73. ESIMS $m / z 285.3$ $\left[\mathrm{M}+\mathrm{H}^{+}\right]$. 
1.3.3. 1-(3'-Deoxy-4',6'-0-isopropylidene-3'-methylene- $\beta$-Dglucopyranosyl)thymine (7a)

To a stirred suspension of $\mathbf{6 a}(5.45 \mathrm{mmol}, 1.55 \mathrm{~g})$ in anhydrous acetone $(93.12 \mathrm{~mL})$ and 2,2-dimethoxypropane $(4.87 \mathrm{~mL})$ was added $p$-toluenesulfonic acid monohydrate $(0.93 \mathrm{mmol}, 0.176 \mathrm{~g})$. After $3 \mathrm{~h}$ the resulting solution was neutralized with triethylamine so that $\mathrm{pH}$ did not exceed 7 . The solution was concentrated and the residue was purified by flash chromatography (4:6 hexane-EtOAc, $R_{\mathrm{f}} 0.26$ ) to give $1.27 \mathrm{~g}(72 \%)$ of $7 \mathrm{a}$ as a white solid: $\mathrm{mp} 167-169^{\circ} \mathrm{C}$ (hexane-EtOAc); $[\alpha]_{\mathrm{D}}^{22}+8\left(\right.$ c $\left.0.5, \mathrm{CHCl}_{3}\right) ; \lambda_{\max }\left(\mathrm{CHCl}_{3}\right) 265 \mathrm{~nm}(\varepsilon$ 9477); ${ }^{1} \mathrm{H}$ NMR $\left(\mathrm{CDCl}_{3}, 400 \mathrm{MHz}\right): \delta 8.31$ (br s, $\left.1 \mathrm{H}, \mathrm{NH}\right), 7.16$ (s, $1 \mathrm{H}, \mathrm{H}-6), 5.51\left(\mathrm{~d}, 1 \mathrm{H}, J_{1^{\prime}, 2^{\prime}} 8.9 \mathrm{~Hz}, \mathrm{H}-1^{\prime}\right), 5.40,5.33$ (2s, 2H, methylene), 4.10-4.04 ( $\left.\mathrm{m}, 2 \mathrm{H}, \mathrm{H}-2^{\prime}, \mathrm{H}-4^{\prime}\right), 3.97-3.76$ ( $\mathrm{m}, 2 \mathrm{H}, \mathrm{H}-6 \mathrm{a}^{\prime}, \mathrm{H}-$ $\left.6 b^{\prime}\right), 3.44-3.36\left(\mathrm{~m}, 1 \mathrm{H}, \mathrm{H}-5^{\prime}\right), 1.91\left(\mathrm{~s}, 3 \mathrm{H}, 5-\mathrm{CH}_{3}\right), 1.56,1.50(2 \mathrm{~s}$, $6 \mathrm{H}, 2 \mathrm{CH}_{3}$ ). Anal. Calcd for $\mathrm{C}_{15} \mathrm{H}_{20} \mathrm{~N}_{2} \mathrm{O}_{6}$ : C, 55.55; $\mathrm{H}, 6.22 ; \mathrm{N}, 8.64$. Found: C, 55.61; H, 6.34; N, 8.58. ESIMS $m / z 325.3\left[\mathrm{M}+\mathrm{H}^{+}\right]$.

\subsubsection{1-(3'-Deoxy-4',6'-0-isopropylidene-3'-methylene- $\beta$-D- glucopyranosyl-2'-ulose)thymine (8a)}

A mixture of 7a $(3.92 \mathrm{mmol}, 1.27 \mathrm{~g})$ and $\mathrm{Ac}_{2} \mathrm{O}(101.53 \mathrm{mmol}$, $9.58 \mathrm{~mL}$ ) was stirred in $15 \mathrm{~mL}$ of dry DMSO/EtOAc (4/1) for $8 \mathrm{~min}$ under nitrogen at $98{ }^{\circ} \mathrm{C}$ and then was concentrated in vacuum. The reaction mixture was extracted with EtOAc, the organic extract was washed with water dried over anhydrous sodium sulfate, filtered, and evaporated to dryness. The residue was purified by flash chromatography ( $1: 1$ hexane-EtOAc, $R_{\mathrm{f}} 0.3$ ) to give $0.61 \mathrm{~g}(48 \%)$ of compound 8a as a white foam, which was found to undergo decomposition during storage: ${ }^{1} \mathrm{H}$ NMR $\left(\mathrm{CDCl}_{3}, 400 \mathrm{MHz}\right): \delta 8.53$ (br s, 1H, NH), 6.97 (s, 1H, H-6), 6.32, 5.83 (2s, 2H, methylene), $5.37\left(\mathrm{~s}, 1 \mathrm{H}, \mathrm{H}-1^{\prime}\right), 4.69\left(\mathrm{~d}, 1 \mathrm{H}, J_{4^{\prime}, 5^{\prime}} 10.0 \mathrm{~Hz}, \mathrm{H}-4^{\prime}\right), 4.00-3.64(\mathrm{~m}$, $\left.3 \mathrm{H}, \mathrm{H}-5^{\prime}, \mathrm{H}-6 \mathrm{a}^{\prime}, \mathrm{H}-6 \mathrm{~b}^{\prime}\right), 1.93\left(\mathrm{~s}, 3 \mathrm{H}, 5-\mathrm{CH}_{3}\right), 1.58,1.49(2 \mathrm{~s}, 6 \mathrm{H}$, $2 \mathrm{CH}_{3}$ ). Anal. Calcd for $\mathrm{C}_{15} \mathrm{H}_{18} \mathrm{~N}_{2} \mathrm{O}_{6}$ : C, 55.90; $\mathrm{H}, 5.63$; N, 8.69. Found: C, 55.69; $\mathrm{H}, 5.37 ; \mathrm{N}, 8.58$. ESIMS $m / z 323.3\left[\mathrm{M}+\mathrm{H}^{+}\right]$.

\subsubsection{1-(3'-Deoxy-3'-methylene- $\beta$-D-glucopyranosyl-2'- ulose)thymine (9a)}

To a solution of $8 \mathbf{a}(1.88 \mathrm{mmol}, 0.61 \mathrm{~g})$ in dry $\mathrm{CH}_{2} \mathrm{Cl}_{2}(3.7 \mathrm{~mL})$ was added methanolic hydrogen chloride $(3.7 \mathrm{~mL})$, which was obtained from acetyl chloride $(0.37 \mathrm{~mL})$ in dry $\mathrm{MeOH}(9.4 \mathrm{~mL})$ at $0{ }^{\circ} \mathrm{C}$. After the mixture was stirred overnight, the solution was concentrated, and the residue was purified by flash chromatography (EtOAc, $\left.R_{\mathrm{f}} 0.35\right)$ to give $0.34 \mathrm{~g}(63 \%)$ of $9 \mathrm{a}$ as a white foam: $[\alpha]_{\mathrm{D}}^{22}$ -26 (c 0.5, $\left.\mathrm{CHCl}_{3}\right) ; \lambda_{\max }\left(\mathrm{CHCl}_{3}\right) 260 \mathrm{~nm}(\varepsilon \quad 7004) ;{ }^{1} \mathrm{H}$ NMR $\left(\left(\mathrm{CD}_{3}\right)_{2} \mathrm{CO}, 400 \mathrm{MHz}\right): \delta 8.51$ (br s, $\left.1 \mathrm{H}, \mathrm{NH}\right), 7.35$ (s, 1H, H-6), 7.21 (br s, $1 \mathrm{H}, \mathrm{H}-1^{\prime}$ ), 6.22 (br s, $1 \mathrm{H}, \mathrm{H}-4^{\prime}$ ), 4.93 (br s, $1 \mathrm{H}, \mathrm{H}-5^{\prime}$ ), 4.11 (s, 2H, methylene), 3.86-3.76 (m, 2H, H-6a', H-6b'), $1.92(\mathrm{~s}, 3 \mathrm{H}$, $\left.5-\mathrm{CH}_{3}\right) ;{ }^{13} \mathrm{C}$ NMR $\left(\left(\mathrm{CD}_{3}\right)_{2} \mathrm{CO}, 100 \mathrm{MHz}\right): 189.1,145.3,141.6$, 135.6, 102.3, 101.5, 82.5, 76.2, 67.6, 63.7, 57.9, 10.9. Anal. Calcd for $\mathrm{C}_{12} \mathrm{H}_{14} \mathrm{~N}_{2} \mathrm{O}_{6}$ : C, 51.06; H, 5.00; N, 9.93. Found: C, 51.34; $\mathrm{H}$, 4.92; N, 9.99. ESIMS $m / z 283.3\left[\mathrm{M}+\mathrm{H}^{+}\right]$.

\subsection{Synthesis of 1-(3'-deoxy-3'-methylene-p-D-glucopyranosyl- $2^{\prime}$-ulose)uracil (9b)}

\subsubsection{1-(2', $4^{\prime}, 6^{\prime}$-Tri-O -acetyl-3'-deoxy-3'-methylene- $\beta$-D- glucopyranosyl)uracil (5b)}

A mixture of uracil (14.00 mmol, $1.57 \mathrm{~g}$ ), HMDS (17.36 mmol, $3.67 \mathrm{~mL})$, and saccharin $(0.64 \mathrm{mmol}, 117.97 \mathrm{mg})$ in dry $\mathrm{CH}_{3} \mathrm{CN}$ $(44 \mathrm{~mL})$ was refluxed for $30 \mathrm{~min}$. To this were added 1,2,4,6-tetra$O$-acetyl-3-deoxy-3-methylene-D-glucopyranose (4), (10.00 $\mathrm{mmol}$, $3.44 \mathrm{~g})$, and $\mathrm{SnCl}_{4}(14.00 \mathrm{mmol}, 1.6 \mathrm{~mL})$. The reaction mixture was refluxed at $80^{\circ} \mathrm{C}$ for $1 \mathrm{~h}$, neutralized with saturated sodium bicarbonate, and then extracted with $\mathrm{CH}_{2} \mathrm{Cl}_{2}$. The organic extract was dried over anhydrous sodium sulfate, filtered, and evaporated to dryness. The residue was purified by flash chromatography
(4:6 hexane-EtOAc, $R_{\mathrm{f}} 0.3$ ) to give $2.1 \mathrm{~g}$ (53\%) of compound $\mathbf{5 b}$, as a white foam: $[\alpha]_{\mathrm{D}}^{22}-2\left(c 0.5, \mathrm{CHCl}_{3}\right) ; \lambda_{\max }\left(\mathrm{CHCl}_{3}\right) 257 \mathrm{~nm}(\varepsilon 6139)$; ${ }^{1} \mathrm{H} \mathrm{NMR}\left(\mathrm{CDCl}_{3}, 400 \mathrm{MHz}\right): \delta 8.08$ (br s, $\left.1 \mathrm{H}, \mathrm{NH}\right), 7.38$ (d, $1 \mathrm{H}, J_{5,6}$ $8.2 \mathrm{~Hz}, \mathrm{H}-6$ ), 5.82 (d, 1H, H-5), 5.67 (d, 1H, J ${1^{\prime}, 2^{\prime}} 9.2 \mathrm{~Hz}, \mathrm{H}-1^{\prime}$ ), 5.48 $\left(\mathrm{d}, 1 \mathrm{H}, \mathrm{H}-2^{\prime}\right), 5.36\left(\mathrm{~d}, 1 \mathrm{H}, J_{4^{\prime}, 5^{\prime}} 9.8 \mathrm{~Hz}, \mathrm{H}-4^{\prime}\right), 5.23,5.12(2 \mathrm{~s}, 2 \mathrm{H}$, methylene), 4.34-4.15 ( $\left.\mathrm{m}, 2 \mathrm{H}, \mathrm{H}-6 \mathrm{a}^{\prime}, \mathrm{H}-6 \mathrm{~b}^{\prime}\right), 3.79-3.77$ ( $\left.\mathrm{m}, 1 \mathrm{H}, \mathrm{H}-5^{\prime}\right), 2.19$, 2.12, 2.10 (3s, 9H, 3OAc). Anal. Calcd for $\mathrm{C}_{17} \mathrm{H}_{20} \mathrm{~N}_{2} \mathrm{O}_{9}$ : C, 51.52; $\mathrm{H}$, 5.09; N, 7.07. Found: C, 51.59; H, 5.27; N, 7.01. ESIMS $m / z 397.4$ $\left[\mathrm{M}+\mathrm{H}^{+}\right]$.

\subsubsection{1-(3'-Deoxy-3'-methylene- $\beta$-D-glucopyranosyl)uracil (6b)}

Uracil derivative $\mathbf{6 b}$ was synthesized from $\mathbf{5 b}$ by the similar procedure as described for $\mathbf{6 a}$. It was purified by flash chromatography (EtOAc, $R_{\mathrm{f}} 0.25$ ) to give $1.3 \mathrm{~g}(90 \%)$ of compound $\mathbf{6 b}$, as a white foam: $[\alpha]_{\mathrm{D}}^{22}+2$ (c $\left.0.1, \mathrm{MeOH}\right) ; \lambda_{\max }(\mathrm{MeOH}) 261 \mathrm{~nm}(\varepsilon$ 7795); ${ }^{1} \mathrm{H}$ NMR $\left(\mathrm{CD}_{3} \mathrm{OD}, 400 \mathrm{MHz}\right): \delta 7.81\left(\mathrm{~d}, 1 \mathrm{H}, J_{5,6} 8.1 \mathrm{~Hz}, \mathrm{H}-\right.$ 6), $5.76(\mathrm{~d}, 1 \mathrm{H}, \mathrm{H}-5), 5.39$ (s, 2H, methylene), $5.36\left(\mathrm{~d}, 1 \mathrm{H}, J_{1^{\prime}, 2^{\prime}}\right.$ $\left.9.2 \mathrm{~Hz}, \mathrm{H}-1^{\prime}\right), 4.23$ (d, 1H, H-2'), 4.10 (d, $\left.1 \mathrm{H}, J_{4^{\prime}, 5^{\prime}} 9.6 \mathrm{~Hz}, \mathrm{H}-4^{\prime}\right)$, 3.92-3.78 (m, 2H, H-6a', H-6b'), 3.33-3.31 (m, $\left.1 \mathrm{H}, \mathrm{H}-5^{\prime}\right) ;{ }^{13} \mathrm{C}$ NMR ( $\left.\mathrm{CD}_{3} \mathrm{OD}, 100 \mathrm{MHz}\right): 153.1,151.2,150.0,142.7,106.3,103.1$, 86.6, 84.1, 71.2, 68.6, 62.7. Anal. Calcd for $\mathrm{C}_{11} \mathrm{H}_{14} \mathrm{~N}_{2} \mathrm{O}_{6}$ : C, 48.89; $\mathrm{H}, 5.22 ; \mathrm{N}, 10.37$. Found: C, 48.85; H, 5.29; N, 10.43. ESIMS $m / z$ $271.3\left[\mathrm{M}+\mathrm{H}^{+}\right]$.

\subsubsection{1-(3'-Deoxy-4',6'-0-isopropylidene-3'-methylene- $\beta$-D- glucopyranosyl)uracil (7b)}

Uracil derivative $\mathbf{7 b}$ was synthesized from $\mathbf{6 b}$ by the similar procedure as described for $\mathbf{7 a}$. It was purified by flash chromatography (EtOAc, $R_{\mathrm{f}} 0.15$ ) to give $1.11 \mathrm{~g}(75 \%)$ of compound $\mathbf{7 b}$ as a white solid: $\mathrm{mp} 179-181^{\circ} \mathrm{C}$ (hexane-EtOAc): $[\alpha]_{\mathrm{D}}^{22}+4($ c 0.5 , $\left.\mathrm{CHCl}_{3}\right) ; \lambda_{\max }\left(\mathrm{CHCl}_{3}\right) 261 \mathrm{~nm} \quad\left(\varepsilon\right.$ 7314); ${ }^{1} \mathrm{H} \quad \mathrm{NMR} \quad\left(\mathrm{CDCl}_{3}\right.$, $400 \mathrm{MHz}$ ): $\delta 8.46$ (br s, $1 \mathrm{H}, \mathrm{NH}), 7.37\left(\mathrm{~d}, 1 \mathrm{H}, J_{5,6} 8.1 \mathrm{~Hz}, \mathrm{H}-6\right)$, $5.76(\mathrm{~d}, 1 \mathrm{H}, \mathrm{H}-5), 5.46\left(\mathrm{~d}, 1 \mathrm{H}, J_{1^{\prime}, 2^{\prime}} 8.9 \mathrm{~Hz}, \mathrm{H}-1^{\prime}\right), 5.34,5.32(2 \mathrm{~s}$, $2 \mathrm{H}$, methylene), $4.14\left(\mathrm{~d}, 1 \mathrm{H}, \mathrm{H}-2^{\prime}\right), 4.04\left(\mathrm{~d}, 1 \mathrm{H}, J_{4^{\prime}, 5^{\prime}} 9.5 \mathrm{~Hz}, \mathrm{H}-4^{\prime}\right)$, 3.96-3.76 (m, 2H, H-6a', H-6b'), 3.39-3.34 (m, 1H, H-5'), 1.55, $1.50\left(2 \mathrm{~s}, 6 \mathrm{H}, 2 \mathrm{CH}_{3}\right)$. Anal. Calcd for $\mathrm{C}_{14} \mathrm{H}_{18} \mathrm{~N}_{2} \mathrm{O}_{6}$ : C, 54.19; $\mathrm{H}, 5.85$; $\mathrm{N}, 9.03$. Found: C, 54.35; $\mathrm{H}, 5.89 ; \mathrm{N}, 9.39$. ESIMS $m / z 311.30\left[\mathrm{M}+\mathrm{H}^{+}\right]$.

\subsubsection{1-(3'-Deoxy-4', $6^{\prime}$-O -isopropylidene-3'-methylene- $\beta$-D- glucopyranosyl-2'-ulose)uracil (8b)}

Uracil derivative $\mathbf{8 b}$ was synthesized from $\mathbf{7 b}$ by the similar procedure as described for $\mathbf{8 a}$. It was purified by flash chromatography ( $1: 1$ hexane-EtOAc $R_{\mathrm{f}} 0.2$ ) to give $0.61 \mathrm{~g}$ (55\%) of compound 8b as a white foam, which was found to undergo decomposition during storage: ${ }^{1} \mathrm{H}$ NMR $\left(\mathrm{CDCl}_{3}, 400 \mathrm{MHz}\right): \delta 8.33$ (br s, $\left.1 \mathrm{H}, \mathrm{NH}\right)$, 7.15 (d, 1H, J5, $7.9 \mathrm{~Hz}, \mathrm{H}-6), 6.32,5.84$ (2s, 2H, methylene), 5.76 (d, $1 \mathrm{H}, \mathrm{H}-5), 5.36\left(\mathrm{~s}, 1 \mathrm{H}, \mathrm{H}-1^{\prime}\right), 4.69\left(\mathrm{~d}, 1 \mathrm{H}, J_{4^{\prime}, 5^{\prime}} 10.0 \mathrm{~Hz}, \mathrm{H}-4^{\prime}\right)$, 4.30-3.64 (m, 3H, H-5', H-6a', H-6b'), 1.59, 1.49 (2s, 6H, 2CH3). Anal. Calcd for $\mathrm{C}_{14} \mathrm{H}_{16} \mathrm{~N}_{2} \mathrm{O}_{6}$ : C, 54.54; $\mathrm{H}, 5.23 ; \mathrm{N}, 9.09$. Found: C, 54.63; $\mathrm{H}, 5.30 ; \mathrm{N}, 9.17$. ESIMS $m / z 309.3\left[\mathrm{M}+\mathrm{H}^{+}\right]$.

\subsubsection{1-(3'-Deoxy-3'-methylene- $\beta$-D-glucopyranosyl-2'- ulose)uracil (9b)}

Uracil derivative $\mathbf{9 b}$ was synthesized from $\mathbf{8 b}$ by the similar procedure as described for 9a. It was purified by flash chromatography (EtOAc, $R_{\mathrm{f}} 0.1$ ) to give $0.32 \mathrm{~g}(60 \%)$ of $\mathbf{9 b}$ as a white foam: $[\alpha]_{\mathrm{D}}^{22}+2(c 0.1, \mathrm{MeOH}) ; \lambda_{\max }(\mathrm{MeOH}) 259 \mathrm{~nm}(\varepsilon 7220) ;{ }^{1} \mathrm{H}$ NMR $\left(\left(\mathrm{CD}_{3}\right)_{2} \mathrm{CO}, 400 \mathrm{MHz}\right): \delta 8.51($ br s, $1 \mathrm{H}, \mathrm{NH}), 7.46\left(\mathrm{~d}, 1 \mathrm{H}, J_{5,6}\right.$ $8.0 \mathrm{~Hz}, \mathrm{H}-6$ ), 7.21 (br s, $1 \mathrm{H}, \mathrm{H}-1^{\prime}$ ), 6.29 (br s, $1 \mathrm{H}, \mathrm{H}-4^{\prime}$ ), 5.65 (d, $1 \mathrm{H}, \mathrm{H}-5), 4.97-4.91\left(\mathrm{~m}, 1 \mathrm{H}, \mathrm{H}-5^{\prime}\right), 4.12$ (br s, $2 \mathrm{H}$, methylene), 3.85-3.81 (m, 2H, H-6a', H-6b'); ${ }^{13} \mathrm{C}$ NMR $\left(\left(\mathrm{CD}_{3}\right)_{2} \mathrm{CO}, 100 \mathrm{MHz}\right)$ : 189.4, 144.8, 142.1, 135.1, 102.2, 100.5, 82.4, 76.4, 67.4, 63.6, 57.8. Anal. Calcd for $\mathrm{C}_{11} \mathrm{H}_{12} \mathrm{~N}_{2} \mathrm{O}_{6}$ : $\mathrm{C}, 49.26 ; \mathrm{H}, 4.51 ; \mathrm{N}, 10.44$. Found: C, 49.33; H, 4.82; N, 10.68. ESIMS $m / z 269.2\left[\mathrm{M}+\mathrm{H}^{+}\right]$. 
1.5. Synthesis of 1 -( $3^{\prime}$-deoxy-3'-methylene- $\beta$-D-glucopyranosyl2'-ulose)5-fluorouracil (9c)

\subsubsection{1-(2',4',6'-Tri-O-acetyl-3'-deoxy-3'-methylene- $\beta$-D- glucopyranosyl)5-fluorouracil (5c)}

A mixture of 5-fluorouracil (13.92 mmol, 1.81 g), HMDS $(17.26 \mathrm{mmol}, 3.64 \mathrm{~mL})$, and saccharin $(0.64 \mathrm{mmol}, 117.29 \mathrm{mg})$ in dry $\mathrm{CH}_{3} \mathrm{CN}(68 \mathrm{~mL})$ was refluxed for $30 \mathrm{~min}$. To this were added 1,2,4,6-tetra-O-acetyl-3-deoxy-3-methylene-D-glucopyranose (4), (10.71 mmol, $3.7 \mathrm{~g}$ ), and $\mathrm{Me}_{3} \mathrm{SiOSO}_{2} \mathrm{CF}_{3}$ (14.99 mmol, $2.7 \mathrm{~mL}$ ). The reaction mixture was refluxed at $65{ }^{\circ} \mathrm{C}$ for $1.5 \mathrm{~h}$, neutralized with saturated sodium bicarbonate, and then extracted with $\mathrm{CH}_{2} \mathrm{Cl}_{2}$. The organic extract was dried over anhydrous sodium sulfate, filtered, and evaporated to dryness. The residue was purified by flash chromatography (4:6 hexane-EtOAc, $R_{\mathrm{f}} 0.2$ ) to give $2.57 \mathrm{~g} \mathrm{(58 \% )} \mathrm{of}$ compound $\mathbf{5 c}$ as a white foam: $[\alpha]_{\mathrm{D}}^{22}+2\left(c 0.3, \mathrm{CHCl}_{3}\right) ; \lambda_{\max }\left(\mathrm{CHCl}_{3}\right)$ $261 \mathrm{~nm}(\varepsilon 7446) ;{ }^{1} \mathrm{H}$ NMR $\left(\mathrm{CDCl}_{3}, 400 \mathrm{MHz}\right): \delta 8.43($ br s, $1 \mathrm{H}, \mathrm{NH})$, $7.46\left(\mathrm{~d}, 1 \mathrm{H}, J_{\mathrm{F} 5,6} 5.5 \mathrm{~Hz}, \mathrm{H}-6\right), 5.64$ (d, 1H, $\left.J_{1^{\prime}, 2^{\prime}} 9.0 \mathrm{~Hz}, \mathrm{H}-1^{\prime}\right), 5.44$ (d,

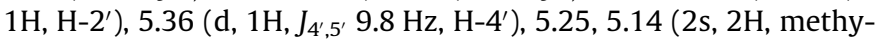
lene), 4.34-4.15 (m, 2H, H-6a', H-6b'), 3.80-3.78 (m, 1H, H-5'), 2.19, 2.13, 2.11 (3s, 9H, 3OAc). Anal. Calcd for $\mathrm{C}_{17} \mathrm{H}_{19} \mathrm{FN}_{2} \mathrm{O}_{9}$ : C, 49.28; H, 4.62; N, 6.76. Found: C, 49.41; H, 4.32; N, 6.83. ESIMS $\mathrm{m} / \mathrm{z} 415.3\left[\mathrm{M}+\mathrm{H}^{+}\right]$.

\subsubsection{1-(3'-Deoxy-3'-methylene- $\beta$-D-glucopyranosyl)5- fluorouracil (6c)}

5 -Fluorouracil analog $\mathbf{6 c}$ was synthesized from $\mathbf{5 c}$ by the similar procedure as described for $\mathbf{6 a}$. The residue was chromatographed (EtOAc, $R_{\mathrm{f}} 0.22$ ) and $1.65 \mathrm{~g}(92 \%)$ of $\mathbf{6 c}$ was obtained as a white foam: $[\alpha]_{\mathrm{D}}^{22}+10\left(c\right.$ 0.7, MeOH); $\lambda_{\max }(\mathrm{MeOH}) 269 \mathrm{~nm}(\varepsilon 6019) ;{ }^{1} \mathrm{H}$ NMR ( $\left.\mathrm{CD}_{3} \mathrm{OD}, 400 \mathrm{MHz}\right): \delta 8.02\left(\mathrm{~d}, 1 \mathrm{H}, J_{\mathrm{F} 5,6} 6.7 \mathrm{~Hz}, \mathrm{H}-6\right), 5.39$ (s, $2 \mathrm{H}$, methylene), 5.35 (d, $\left.1 \mathrm{H}, J_{1^{\prime}, 2^{\prime}} 9.1 \mathrm{~Hz}, \mathrm{H}-1^{\prime}\right), 4.20$ (d, $1 \mathrm{H}, \mathrm{H}-2^{\prime}$ ), $4.11\left(\mathrm{~d}, 1 \mathrm{H}, J_{4^{\prime}, 5^{\prime}} 11.5 \mathrm{~Hz}, \mathrm{H}-4^{\prime}\right), 3.92-3.77\left(\mathrm{~m}, 2 \mathrm{H}, \mathrm{H}-6 \mathrm{a}^{\prime}, \mathrm{H}-6 \mathrm{~b}^{\prime}\right)$, 3.31-3.28 (m, $\left.1 \mathrm{H}, \mathrm{H}-5^{\prime}\right)$; ${ }^{13} \mathrm{C}$ NMR $\left(\mathrm{CD}_{3} \mathrm{OD}, 100 \mathrm{MHz}\right): 150.2$, 149.0, 132.3, 131.7, 128.6, 105.2, 85.7, 82.5, 69.6, 67.2, 61.2. Anal. Calcd for $\mathrm{C}_{11} \mathrm{H}_{13} \mathrm{FN}_{2} \mathrm{O}_{6}$ : C, 45.84; $\mathrm{H}, 4.55 ; \mathrm{N}, 9.72$. Found: $\mathrm{C}$, 45.11; $\mathrm{H}, 4.27$; $\mathrm{N}, 9.38$. ESIMS $m / z 289.3\left[\mathrm{M}+\mathrm{H}^{+}\right]$.

\subsubsection{1-(3'-Deoxy-4',6'-O-isopropylidene-3'-methylene- $\beta$-D- glucopyranosyl)5-fluorouracil (7c)}

5 -Fluorouracil analog $7 \mathbf{c}$ was synthesized from $\mathbf{6 c}$ by the similar procedure as described for $7 \mathbf{a}$. It was purified by flash chromatography (6:4 hexane-EtOAc, $R_{\mathrm{f}} 0.2$ ) to give $1.27 \mathrm{~g}(68 \%)$ of compound 7c as a white foam: $[\alpha]_{\mathrm{D}}^{22}+8\left(\right.$ c $\left.0.4, \mathrm{CHCl}_{3}\right)$; $\mathrm{UV} \lambda_{\max }\left(\mathrm{CHCl}_{3}\right) 267 \mathrm{~nm}$ ( $\varepsilon$ 6018); ${ }^{1} \mathrm{H} \mathrm{NMR}\left(\mathrm{CDCl}_{3}, 400 \mathrm{MHz}\right): \delta 9.64(\mathrm{br} \mathrm{s}, 1 \mathrm{H}, \mathrm{NH}), 7.44(\mathrm{~d}$, $\left.1 \mathrm{H}, J_{\mathrm{F} 5,6} 5.3 \mathrm{~Hz}, \mathrm{H}-6\right), 5.48$ (d, 1H, $\left.J_{1^{\prime}, 2^{\prime}} 8.8 \mathrm{~Hz}, \mathrm{H}-1^{\prime}\right), 5.37,5.35$ (2s, $2 \mathrm{H}$, methylene), $4.11\left(\mathrm{~d}, 1 \mathrm{H}, \mathrm{H}-2^{\prime}\right), 4.04\left(\mathrm{~d}, 1 \mathrm{H}, J_{4^{\prime}, 5^{\prime}} 9.4 \mathrm{~Hz}, \mathrm{H}-4^{\prime}\right)$, 3.96-3.77 (m, 2H, H-6a', H-6b'), 3.42-3.37 (m, 1H, H-5'), 1.55, $1.49\left(2 \mathrm{~s}, 6 \mathrm{H}, 2 \mathrm{CH}_{3}\right)$. Anal. Calcd for $\mathrm{C}_{14} \mathrm{H}_{17} \mathrm{FN}_{2} \mathrm{O}_{6}: \mathrm{C}, 51.22 ; \mathrm{H}$, 5.22 ; N, 8.53. Found: C, 51.29; H, 5.41; N, 8.47. ESIMS $m / z 329.3$ $\left[\mathrm{M}+\mathrm{H}^{+}\right]$.

\subsubsection{1-(3'-Deoxy-4',6'-O -isopropylidene-3'-methylene- $\beta$-D- glucopyranosyl-2'-ulose)5-fluorouracil (8c)}

5 -Fluorouracil analog $8 \mathbf{c}$ was synthesized from $7 \mathbf{c}$ by the similar procedure as described for $\mathbf{8 a}$. Chromatography of the residue $(1: 1$ hexane-EtOAc, $\left.R_{\mathrm{f}} 0.45\right)$ gave $0.58 \mathrm{~g}(46 \%)$ of compound $8 \mathrm{c}$ as a white foam, which was found to undergo decomposition during storage: ${ }^{1} \mathrm{H}$ NMR $\left(\mathrm{CDCl}_{3}, 400 \mathrm{MHz}\right): \delta 9.18$ (br s, $\left.1 \mathrm{H}, \mathrm{NH}\right), 7.37$ (d, $\left.1 \mathrm{H}, J_{\mathrm{F} 5,6} 5.3 \mathrm{~Hz}, \mathrm{H}-6\right), 6.32,5.83$ (2s, 2H, methylene), $5.32(\mathrm{~m}, 1 \mathrm{H}$, $\left.\mathrm{H}-1^{\prime}\right), 4.67$ (d, 1H, $\left.J_{4^{\prime}, 5^{\prime}} 10.0 \mathrm{~Hz}, \mathrm{H}-4^{\prime}\right), 4.24-3.66$ (m, 3H, H-5', H$\left.6 \mathrm{a}^{\prime}, \mathrm{H}-6 \mathrm{~b}^{\prime}\right), 1.59,1.51\left(2 \mathrm{~s}, 6 \mathrm{H}, 2 \mathrm{CH}_{3}\right)$. Anal. Calcd for $\mathrm{C}_{14} \mathrm{H}_{15} \mathrm{FN}_{2} \mathrm{O}_{6}$ : C, 51.54; H, 4.63; N, 8.59. Found: C, 51.69; H, 4.74; N, 8.89. ESIMS $\mathrm{m} / \mathrm{z} 327.3\left[\mathrm{M}+\mathrm{H}^{+}\right]$.
1.5.5. 1-(3'-Deoxy-3'-methylene- $\beta$-D-glucopyranosyl-2'-ulose)5fluorouracil (9c)

5-Fluorouracil derivative 9c was synthesized from $\mathbf{8 c}$ by the similar procedure as described for 9a. It was purified by flash chromatography (0.5: $\left.9.5 \mathrm{MeOH}-\mathrm{CH}_{2} \mathrm{Cl}_{2}, R_{\mathrm{f}} 0.35\right)$ to give $0.28 \mathrm{~g}(55 \%)$ of compound 9c as a white foam: $[\alpha]_{\mathrm{D}}^{22}-26$ (c 0.4, MeOH); $\lambda_{\max }$ $(\mathrm{MeOH}) 262 \mathrm{~nm}\left(\varepsilon\right.$ 9525); ${ }^{1} \mathrm{H}$ NMR $\left(\left(\mathrm{CD}_{3}\right)_{2} \mathrm{CO}, 400 \mathrm{MHz}\right): \delta 7.73$ (d, $\left.1 \mathrm{H}, J_{\mathrm{F} 5,6} 6.6 \mathrm{~Hz}, \mathrm{H}-6\right), 7.21$ (br s, $1 \mathrm{H}, \mathrm{H}-1^{\prime}$ ), 6.31 (br s, $1 \mathrm{H}, \mathrm{H}-$ $4^{\prime}$ ), 4.98 (br s, $\left.1 \mathrm{H}, \mathrm{H}-5^{\prime}\right), 4.12$ (s, 2H, methylene), 3.88-3.84 (m, $\left.2 \mathrm{H}, \mathrm{H}-6 \mathrm{a}^{\prime}, \mathrm{H}-6 \mathrm{~b}^{\prime}\right) ;{ }^{13} \mathrm{C}$ NMR $\left(\left(\mathrm{CD}_{3}\right)_{2} \mathrm{CO}, 100 \mathrm{MHz}\right): 189.2,145.1$, 134.6, 127.1, 108.1, 103.3, 82.1, 76.6, 67.9, 63.7, 58.0. Anal. Calcd for $\mathrm{C}_{11} \mathrm{H}_{11} \mathrm{FN}_{2} \mathrm{O}_{6}$ : C, 46.16; $\mathrm{H}, 3.87 ; \mathrm{N}, 9.79$. Found: $\mathrm{C}, 46.25 ; \mathrm{H}$, 3.89; N, 9.86. ESIMS $m / z$ 287.2 [M+H $\left.{ }^{+}\right]$.

\subsection{Cytostatic activity assays}

Murine leukemia L1210, human lymphocyte CEM, and human cervix carcinoma HeLa cells were seeded in 96-well microtiter plates at 50,000 (L1210), 75,000 (CEM) or 20,000 (HeLa) cells per $200 \mu \mathrm{L}$-well in the presence of different concentrations of the test compounds. After 2 (L1210), 3 (CEM) or 4 (HeLa) days, the viable cell number was counted using a Coulter counter apparatus. The $50 \%$ cytostatic concentration $\left(\mathrm{CC}_{50}\right)$ was defined as the compound concentration required to inhibit tumor cell proliferation by $50 \%$.

\section{Acknowledgments}

This work was supported in part by the Postgraduate Programmes 'Biotechnology-Quality assessment in Nutrition and the Environment', 'Application of Molecular Biology-Molecular Genetics-Molecular Markers', Department of Biochemistry and Biotechnology, University of Thessaly, and by the K. U. Leuven (GOA 10/014). We thank Mrs. Lizette van Berckelaer for excellent technical assistance.

\section{References}

1. Zhou, W.; Gumina, G.; Chong, Y.; Wang, J.; Schinazi, R. F.; Chu, C. K. J. Med. Chem. 2004, 47, 3399-3408.

2. Perigaud, C.; Gosselin, G.; Imbach, J. L. Nucleosides Nucleotides 1992, 11, 903 945.

3. Robins, R. K.; Kini, G. D. Purines and Purine Nucleoside Analogs as Antitumor Agents. In The Chemistry of Antitumor Agents; Wilman, D. E. V., Ed.; Chapman and Hall: New York, 1990; pp 229-321.

4. Komiotis, D.; Manta, S.; Tsoukala, E.; Tzioumaki, N. Curr. Med. Chem. Anti-Infect. Agents 2008, 7, 219-244.

5. Van Rompay, A. R.; Johansson, M.; Karlsson, A. Pharmacol. Ther. 2003, 100, 119139.

6. De Clercq, E. Biochim. Biophys. Acta 2002, 1587, 258-275.

7. Paterson, J.; Uriel, C.; Egron, M. J.; Herscovici, J.; Antonakis, K.; Alaoui, M. Antimicrob. Agents Chemother. 1998, 42, 779-784.

8. Antonakis, K.; Halmos, T.; Bach, J.; Chouroulinkov, I. Eur. J. Med. Chem. 1980, 15, 237-240.

9. Verheggen, I.; Van Aerschot, A.; Toppet, S.; Snoeck, R.; Janssen, G.; Balzarini, J.; De Clercq, E.; Herdewijn, P. J. Med. Chem. 1993, 36, 2033-2040.

10. Verheggen, I.; Van Aerschot, A.; Van Meervelt, L.; Rozenski, J.; Wiebe, L.; Snoeck, R.; Andrei, G.; Balzarini, J.; Claes, P.; De Clercq, E.; Herdewijn, P. J. Med. Chem. 1995, 38, 826-835.

11. Ostrowski, T.; Wroblowski, B.; Busson, R.; Rozenski, J.; De Clercq, E.; Bennet, M S.; Champness, J. N.; Summers, W. C.; Sanderson, M. R.; Herdewijn, P. J. Med. Chem. 1998, 41, 4343-4353.

12. Maurinsh, Y.; Schraml, J.; De Winter, H.; Blaton, N.; Peeters, O.; Lescrinier, E.; Rozenski, J.; Van Aerschot, A.; De Clercq, E.; Busson, R.; Herdewijn, P. J. Org. Chem. 1997, 62, 2861-2871.

13. Manta, S.; Tsoukala, E.; Tzioumaki, N.; Goropevšek, A.; Pamulapati, R. T.; Cencič, A.; Balzarini, J.; Komiotis, D. Eur. J. Med. Chem. 2009, 44, 2696-2704.

14. Spanou, C.; Manta, S.; Komiotis, D.; Dervishi, A.; Kouretas, D. Int. J. Mol. Sci. 2007, 8, 695-704.

15. Haouz, A.; Vanheusden, V.; Munier-Lechman, H.; Froeyen, M.; Herdewijn, P.; Van Galenbergh, S.; Delarue, M. J. Biol. Chem. 2003, 278, 4963-4971.

16. Balatsos, N. A. A.; Vlachakis, D.; Maragozidis, P.; Manta, S.; Anastasakis, D.; Kyritsis, A.; Vlassi, M.; Komiotis, D.; Stathopoulos, C. Biochemistry 2009, 48, 6044-6051. 
17. Tsirkone, V. G.; Tsoukala, E.; Lamprakis, C.; Manta, S.; Hayes, J. M.; Skamnaki, V. T.; Drakou, C.; Zographos, S. E.; Komiotis, D.; Leonidas, D. D. Bioorg. Med. Chem. 2010, 18, 3423-3425.

18. Vastmans, K.; Pochet, S.; Peys, A.; Kerremans, L.; Van Aerschot, A.; Hendrix, C. Marliere, P.; Herdewijn, P. Biochemistry 2000, 39, 12757-12765.

19. Vastmans, K.; Froeyen, M.; Kerremans, L.; Pochet, S.; Herdewijn, P. Nucleic Acids Res. 2001, 29, 3154-3163.

20. Herdewijn, P.; De Clercq, E. Bioorg. Med. Chem. Lett. 2001, 11, 1591-1597.

21. Manta, S.; Agelis, G.; Botić, T.; Cencič, A.; Komiotis, D. Bioorg. Med. Chem. 2007, 15, 980-987.

22. Manta, S.; Agelis, G.; Botić, T.; Cencič, A.; Komiotis, D. Eur. J. Med. Chem. 2008 43, 420-428.

23. Manta, S.; Tsoukala, E.; Tzioumaki, N.; Kiritsis, C.; Balzarini, J.; Komiotis, D. Bioorg. Chem. 2010, 38, 48-55.

24. Manta, S.; Tzioumaki, N.; Tsoukala, E.; Panagiotopoulou, A.; Pelecanou, M.; Balzarini, J.; Komiotis, D. Eur. J. Med. Chem. 2009, 44, 4764-4771.

25. Agelis, G.; Tzioumaki, N.; Botić, T.; Cencič, A.; Komiotis, D. Bioorg. Med. Chem. 2007, 15, 5448-5456.

26. Agelis, G.; Tzioumaki, N.; Tselios, T.; Botić, T.; Cencič, A.; Komiotis, D. Eur. J. Med. Chem. 2008, 43, 1366-1375.

27. Tzioumaki, N.; Tsoukala, E.; Manta, S.; Agelis, G.; Balzarini, J.; Komiotis, D. Arch. Pharm. 2009, 342, 353-360.

28. Tzioumaki, N.; Manta, S.; Tsoukala, E.; Voorde, J. V.; Liekens, S.; Komiotis, D. Balzarini, J. Eur. J. Med Chem., submitted for publication.

29. Chirakul, P.; Sigurdsson, S. T. Org. Lett. 2003, 5, 917-919.

30. Liu, X.; Guo, X.; Wang, F.; Lei, G.; Luo, D. Nucleosides Nucleotides Nucleic Acids 2007, 26, 45-49.

31. Elhalabi, J.; Rice, K. G. Nucleosides Nucleotides Nucleic Acids 2004, 23, 195-205.

32. Yoo, S. J.; Kim, H. O.; Lim, Y.; Kim, J.; Jeong, L. S. Bioorg. Med. Chem. 2002, 10, 215-226.
33. Acton, E. M.; Goerner, R. N.; Uh, H. S.; Ryan, K. J.; Henry, D. W.; Cass, C. E.; LePage, G. A. J. Med. Chem. 1979, 22, 518-525.

34. Tsoukala, E.; Agelis, G.; Dolinsek, J.; Botić, T.; Cencič, A.; Komiotis, D. Bioorg. Med. Chem. 2007, 15, 3241-3247.

35. Vanheusden, V.; Busson, R.; Herdewijn, P.; Van Calenbergh, S. J. Org. Chem. 2004, 69, 4446-4453.

36. Pfitzner, K. E.; Moffatt, J. G. J. Am. Chem. Soc. 1965, 87, 5661-5670.

37. Cheng, X.; Zhang, J.-D.; Zhang, L.-H. Synthesis 1989, 383-384.

38. Cook, A. F.; Moffatt, J. G. J. Am. Chem. Soc. 1967, 89, 2697-2705.

39. Garegg, P. J.; Samuelsson, B. Carbohydr. Res. 1978, 67, 267-270.

40. Hansske, F.; Madej, D.; Robins, M. J. Tetrahedron 1984, 40, 125-135.

41. Omura, K.; Swern, D. Tetrahedron 1978, 34, 1651-1660.

42. Perlman, M. E. Nucleosides Nucleotides 1993, 12, 73-82.

43. Ueda, T.; Shuto, S.; Satoh, M.; Inoue, H. Nucleosides Nucleotides 1985, 4, 401409.

44. Serafinowski, P. J.; Barnes, C. L. Tetrahedron 1996, 52, 7929-7938.

45. Dess, D. B.; Martin, J. C. J. Org. Chem. 1983, 48, 4155-4156.

46. Albright, J. D.; Goldman, L. J. Org. Chem. 1965, 30, 1107-1110.

47. Ergon, M. J.; Leclercq, F.; Antonakis, K.; Bennani-Baiti, I.; Frayssinet, C. Carbohydr. Res. 1993, 248, 143-150.

48. Van Rijsbergen, R.; Anteunis, M. J. O.; De Bruyn, A. J. Carbohydr. Chem. 1983, 2, 395-404.

49. Kim, M. J.; Jeong, L. S.; Kim, J. H.; Shin, J. H.; Chung, S. Y.; Lee, S. K.; Chun, M. W. Nucleosides Nucleotides Nucleic Acids 2004, 23, 715-724.

50. Witczak, Z. J.; Lorchak, D.; Nguyen, N. Carbohydr. Res. 2007, 342, 19291933.

51. Szarek, W. A.; Jewell, J. S.; Szczerek, I.; Jones, J. K. N. Can. J. Chem. 1969, 47, 4473-4476.

52. Rosenthal, A.; Sprinzl, M. Can. J. Chem. 1969, 47, 3941-3946. 